\title{
Network Disruptions and the Security of Supply in the European Gas Network
}

\author{
László Á. Kóczy ${ }^{1,2}$, Dávid Csercsik ${ }^{3}$, and Balázs R. Sziklai ${ }^{1,4}$ \\ ${ }^{1}$ Centre for Economic and Regional Studies \\ ${ }^{2}$ Budapest University of Technology and Economics \\ ${ }^{3}$ Pázmány Péter Catholic University \\ ${ }^{4}$ Corvinus University of Budapest
}

\begin{abstract}
The natural gas supply of the Eastern European countries located between Germany and Russia has always been problematic. A historical reliance on Russia has been eased by increased connectivity to the West. Recent developments may lead to a diversified source but via a single supply route, which, in the light of the 2017 Baumgarten explosion, may introduce another type of risk.

We propose a novel framework to measure the supply security of natural gas networks, combining a linear programming approach with a risk assessment technique borrowed from finance which measures supply security in European countries. The expected shortfall (ES) is currently the best practice for risk measurement recommended by one of the most important international financial regulators, the Basel Committee on Banking Supervision. ES assigns risks by looking at a fraction of the worst cases of supply disruptions. The latter is modelled as the change in the optimal network flow in the case of one of the pipelines falling out due to certain incidents.

Several network configurations are considered along with seasonal scenarios corresponding to the difference in the availability of gas from storage facilities. We find that the construction of Nord Stream 2, that is, doubling the capacity of the direct connection between Russia and Germany alleviates the gas shortage problem if the connection via Ukraine
\end{abstract}


is shut down, otherwise, the construction increases the risk for Eastern

Europe.

JEL codes: C71, D72

Keywords and phrases:

\section{Introduction}

Amidst an increasing trend of renewables in energy production, natural gas remains a versatile, widely accepted energy source. Gas-fuelled power stations are flexible, making them ideal as reserves to smooth out demand shocks or complement supply shocks in variable renewables. Due to its role in household heating, any shortage is extremely noticeable and has an immediate impact.

Natural gas is transported via pipelines or ships in liquefied form (LNG) which must be regasified to enter the pipeline network. Both LNG ports and pipelines require substantial investments and, consequently, the pipeline network suffers from congestion problems: some regions may experience supply constraints or must be supplied via long, expensive detours in the network. Congestion problems are especially pronounced in winter periods as natural gas is used extensively for heating and many countries have established storage facilities to smoothen out demand seasonality and alleviate demand shocks or transport disruptions.

Our interest is in the European gas network, characterised by a high reliance on gas from Russia with additional supply from gas fields in the North Sea (Norway), North Africa, Central Asia and - increasingly - LNG to meet demand. We focus on developments in Eastern Europe. Until the opening of Nord Stream in 2011, most gas from Russia was transported via Ukraine. After the escalation of the Russia-Ukraine gas disputes that eventually resulted in gas delivery disruptions to several European countries, Russia started to look for alternative delivery routes bypassing Ukraine. Nord Stream, opened in 2011, together with its nearly complete capacity extension Nord Stream 2 is now able to replace the Ukrainian pipelines in terms of transmission capacity. Russia's main partner, Germany is surely a winner of these developments for getting its energy via a direct connection. Countries in the Eastern ends of the European Union 
(Poland, Slovakia, Hungary, as well as countries on the Balkans), have made substantial progress in diversifying their sourcing and building interconnectors (Tarnawski 2015). Now, with the prospect of Ukrainian pipelines phased out, they must prepare for the situation where, once again, all their energy supply comes via a single pipeline. With one difference: the Brotherhood pipeline carried gas for Germany and other Western European countries so disruptions set major powers into motion, while the prospect is to be at the end of a pipeline that crosses half of Europe. Intuitively, incidents such as the 2017 Baumgarten blast shall effect the region more drastically.

Ours is not the first paper to study supply security (or security of supply, abbreviated as $S O S$ ) and transport security in particular. Well before the 2009 Russia-Ukraine gas dispute, Weisser (2007) put out a manifesto warning of the risk of limited sourcing and a possible shock comparable to the Oil Crisis, calling for action. Despite efforts to increase connectivity and regulate the market, the interest in the supply security of natural gas to various markets remains (Cabalu, 2010, Doukas et al. 2011, Biresselioglu et al., 2015, Lu et al., 2016). These studies take many different aspects into account. Cabalu (2010) considers gas supply interruptions, volatile gas prices, transportation and distribution bottlenecks and a growing reliance on long-distance imports, exploring the vulnerability of certain Asian countries. Stern (2002) distinguishes between source-, transit- and facility-dependence as the main sources of risks in an import-dependent country.

The risks caused by source or facility-dependence include strikes by Norwegian platform workers (World Gas Report, 1986, Adomaitis and Solsvik, 2020), a bombing of an on-shore section a pipeline in Algeria (Energy Compass, 1997) causing long-term concerns of similar attacks, the shutting down of the Arun liquefaction plant in Indonesia for several months due to political instability (Gas Matters, 2001), the 2017 Baumgarten incident (Vainio, 2017) that led Italy to declare a state of emergency (Hassel and Egenhofer, 2017, Bartelet and Mulder, 2020 and the general political and commercial risks of trading with certain countries.

For transit-dependence, Stern (2002) already mentions the case of Russian transits via Ukraine but the 2009 January crisis affected the supply of 18 countries, causing more severe outages than any gas dispute before (Pirani et al. 
2009) and still causing concern as tensions continue.

Our approach is closest to that of Scotti and Vedres $(2012)$ and Praks et al. (2015) in focusing on the network, rather than just the availability of sources. The effect of disruptions in the transportation network is scrutinised using the simplified model of the pipeline network (Csercsik, Hubert, Sziklai, and Kóczy, 2019) and an intuitive model to determine the optimal flows assuming rational payoff-maximising agents. We examine the exposure of different countries to transport disruptions as well as the effect of network developments thereon: beyond a baseline scenario, considering a closing of the Ukrainian corridor, the completion of Nord Stream 2 as well as a combined scenario. These are currently the most neuralgic points in the European network development scene, see e.g. (Jirušek, 2020, Tóth et al., 2020, Sziklai et al., 2020, Wood and Henke, 2021). We include the Trans-Anatolian and Trans-Adriatic Pipelines (TANAP and TAP) in the analysis; the more recent TurkStream and Balkan Stream are not present in our data set yet.

Network disruptions may affect countries in different ways: resulting in a small price increase - a positive effect is uncommon - or even a massive shortage. We assume that shortages can be supplied from storage facilities when these are full - at the end of the summer - but storage facilities must be refilled before the winter months when consumption may exceed supply. If the incident happens in the winter when storages are depleted, any shortage must be compensated by diverting consumption to other energy sources at substantial costs. We refer to these cases as the summer and winter scenarios.

While several indicators have been introduced for supply security for natural gas and energy in general (Kruyt et al., 2009, Vianello and Maschio, 2014, Pavlović et al. 2018), our approach and indicator is new. The expected shortfall became the standard risk measure in finance dethroning value-at-risk (VaR), after some weaknesses have been identified in VaR analyses including the measure's inability to capture 'tail risk' (Basel Committee on Banking Supervision, 2013). Apart from bearing the recommendation of the Basel Committee, it also has sound theoretical foundations (Acerbi, 2002, Adam et al. 2008).

The structure of the paper is as follows. First, we introduce our model, elucidating on its limitations. Next, we discuss the data collected and finally 
present and discuss the main findings.

\section{Model}

In the absence of disruptions, calculating the gas supply of a country or region is a simple optimisation task, where gas is purchased from the available sources taking prices, transportation costs and capacity constraints into account. First, this optimisation problem is described along with the assumptions made. Then our notion of supply security is explained clarifying the winter and summer scenarios. We end the section with an example.

\section{$2.1 \quad$ Notation}

The international natural gas pipeline network is examined to identify countries with nodes and pipelines with the arcs. The set of nodes is denoted by $N$, $|N|=n$ with a generic element denoted by $i$ or $j$. The set of arcs is denoted by $L,|L|=m$ with the generic element $l$. The network itself is described by an incidence matrix $A \in \mathbb{R}^{n \times m}$ with $A_{i l}=-1$ and $A_{j l}=1$ indicating that the pipeline $l$ runs from node $i$ to $j$.

Each node is either a net producer or net consumer: in case of the former, the supply exceeds the local demand. Net consumers supply their demand from the network, from storage or, if these are not possible, switch to other energy sources or reduce their consumption. As a simplification, we assume that there is an indigenous, alternative energy source at each node that is more expensive than any of the natural gas sources including transportation costs and, therefore, only chosen to overcome shortages. This alternative is not necessarily a real energy source, it may also represent the economic losses generated by the gas shortage. This approach has been used by Sziklai, Kóczy, and Csercsik (2020) as well.

Formally, we assume that each node is characterised by demand and production, $d^{0} \in \mathbb{R}_{+}^{n}$ denotes the vector of demands and $s^{0} \in \mathbb{R}_{+}^{n}$ the vector of (maximum) supplies. In the following, we modify these vectors to get $d, s \in \mathbb{R}_{+}^{n}$ : In net producer countries, production is reduced by the local consumption while for net consumers, we add the alternative energy source up to the level of the 
net demand. Therefore,

$$
d_{i}=\left(d_{i}^{0}-s_{i}^{0}\right)^{+} \quad \text { and } \quad s_{i}=\left|d_{i}^{0}-s_{i}^{0}\right|
$$

where $(\cdot)^{+}$denotes the positive part, that is $(x)^{+}=\max \{x, 0\}$ for any $x \in \mathbb{R}$. The production cost is denoted by $p \in \mathbb{R}_{+}^{n}$ where $p_{i}=\bar{p}$ for all $i$ such that $d_{i}^{0}>s_{i}^{0}$, where $\bar{p}$ is the cost of the alternative energy source.

The edges, representing the pipelines, are characterised by maximal transmission capacities in each direction, $q=\left(q^{+}, q^{-}\right) \in \mathbb{R}_{+}^{2 \times m}$. The transmission capacity differences are monitored, mostly for technical reasons; while most pipelines are directed with a reduced reverse flow capacity, changing the direction is neither costly nor particularly time consuming. Given a bidirectional pipeline $l$ and a flow $f_{l}$ on it, the net capacity in the direction of the flow is $q_{l}^{+}-f_{l}$, while in the opposite direction, there is a capacity $q_{l}^{-}+f_{l}$, where the last term represents a potential for barters without actual gas transmission.

Transporting gas over these pipelines has its costs. A pipeline may travel across several regions, making it convenient to define costs by a cost matrix $C \in \mathbb{R}_{+}^{n \times m}$ where $C_{i j}$ is the cost of transferring a unit gas over pipeline $j$ occurring in region $i$. We assume that transportation cost is proportional to the length of the pipeline.

\subsection{Optimal flows}

Optimal flow is one that satisfies the demand while incurring the lowest total cost. By our assumption of utilising alternative energy sources, satisfying demands locally is always a feasible solution.

Formally, let $f_{j}^{+} \in \mathbb{R}_{+}$denote the flow in the positive direction over edge $j$. Similarly, let $f_{j}^{-} \in \mathbb{R}_{+}$denote flow in the opposite direction. Let $I \in \mathbb{R}_{+}^{n}$ denote the inlet values at the nodes. The variable vector is then

$$
x=\left(\begin{array}{c}
f^{+} \\
f^{-} \\
I
\end{array}\right) \in \mathbb{R}_{+}^{2 m+n} .
$$

Let $e^{i}$ and $e^{S}$ denote $n$-dimensional indicator vectors for $i$ and $S$, respec- 
tively:

$$
e_{k}^{i}=\left\{\begin{array}{ll}
1 & \text { if } k=i \\
0 & \text { otherwise }
\end{array} \text { and } e_{k}^{S}= \begin{cases}1 & \text { if } k \in S \\
0 & \text { otherwise }\end{cases}\right.
$$

Let $E^{S}$ denote a diagonal matrix with $e^{S}$ over the diagonal, $1_{n}$ denote an $n$ dimensional vector of 1 's, $I^{k \times k}$ a $k$ dimensional identity matrix and $0^{k \times l}$ denote a $k \times l$-dimensional 0 matrix.

We now describe the linear programming problem and interpret the constraints, we minimise the cost of supplying player $i$ :

$$
\min _{x}\left(\begin{array}{lll}
1_{n}^{T} C & 1_{n}^{T} C & p^{T}
\end{array}\right) x
$$

such that

$$
\begin{aligned}
& {\left[\begin{array}{lll}
A & A & E^{S}
\end{array}\right] x=d_{i} e^{i}} \\
& I_{j} \leq s_{j} \\
& \left(\begin{array}{ll}
I^{2 m \times 2 m} & 0^{2 m \times n}
\end{array}\right) x \leq\left(\begin{array}{c}
q^{+} \\
q^{-}
\end{array}\right) \\
& x \geq 0
\end{aligned}
$$

The objective function and constraints are, actually, rather straightforward. The aim is to minimise the total cost, that is, the sum of the transportation costs in the positive direction, transportation costs in the negative direction and the cost of gas itself. Since the transportation costs are non-zero on each pipeline, gas cannot flow in both directions as it would result in a cheaper flow with the same resulting supply. The first constraint explains that no gas is lost at any of the nodes: the total of inlets, inflows and outflows must add up to the consumption of player $i$. Inlets cannot exceed the supply capacities. The last condition merely insists on the non-negativity of flows. Finally, constraint 2.6 explains that the flows must not exceed the transmission capacities. Initially, we set $q^{+}=q^{-}=q$.

While this simple optimisation problem helps us find the optimal paths, acquiring the individual payoffs must also be considered. Obtaining these payoffs is nontrivial when different supply paths - with different generalised prices intersect. We assume that when gas flows merge the price becomes the weighted 
average of the prices, where weights corresponds to the different volumes of the flows. Since the optimal flows constitute a directed acyclic graph, circular flows could be eliminated to reduce costs - this calculation is well-defined. In Section 2.5, we demonstrate how individual payoffs can be calculated in a detailed example.

\subsection{Supply security}

Out of the many factors that affect supply security, our focus lies on the aspects related to the physical network: on disruptions that put one of the pipelines out of use, including political risks, natural disasters, technical failures but also sabotage and terrorism. In order to evaluate such risks, we turn to models used in finance and the study and the design of electric power grids.

While both are utility networks, the physical properties of the electric power grids are quite different. There are complex laws that determine the flow of electricity depending on the network characteristics, inputs and outputs and the electricity cannot be re-routed to reduce the load on a particular connection. In case of a power-line failure, electricity re-routes itself, increasing the chance of an overload on one of the other connections. Unless the network is protected against such primary failures, we may expect a sequence of problems resulting in widespread and long-term disruptions.

The so-called $N-1$ contingency analysis is used to evaluate the would-be scenario of an incident where one of the power lines drop out (double incidents have a negligible probability). Majidi-Qadikolai and Baldick (2016) explains how $N-1$ contingency analysis can be applied in the efficient planning of the power grid to avoid cascading failures after an incident.

Clearly, in the case of natural gas networks, such preparation is unnecessary: if one of the pipelines is closed down, the closure does not directly affect the operation of other connections, except that congestions may be more severe.

For the nodes of the network, that is, the countries, the effects may prove to be more drastic. In the optimal flow supply is redirected to the remaining routes, increasing congestion along the affected pipelines. As a result, the already limited transmission capacities are further reduced and some countries may find themselves without available gas sources. While a disruption increases the 
overall cost of supplying the network, players may be hardly or severely affected depending upon their connectedness. In rare cases, a player may even benefit from the change (we will show how this can happen in the example network).

Our analysis covers all possible incidents, that is, scenarios corresponding to the closure of each of the existing pipelines. We assume that each pipeline fails with the same probability leading to a random variable that informs the extent to which each country will be hit in case of an incident. Our method can be generalised to accommodate pipeline-specific risks.

We evaluate these scenarios using a risk measure (Denault, 2001, Szegö 2002 ) which is the (possibly negative) amount of cash that needs to be added to a problem to make it acceptable and is calculated as a weighted average of all the scenarios. We express the conservatism of the players by assigning higher weights to more critical scenarios, in other words, we use spectral risk measures (Acerbi 2002). Specifically, we employ the $\alpha$-expected shortfall with $\alpha=10 \%$, the most popular spectral risk measure in economics and finance. The expected shortfall takes the (weighted) average of the worst $10 \%$ of scenarios; in our case, this refers to inspecting the disruptions that affect the player least favourably.

\subsection{Seasonality}

The European natural gas network exhibits a natural seasonality. As the gas is used for heating, among other services, consumption is higher in the winter than in the summer. In fact, transmission capacities are often insufficient to cater to winter demands: storage is filled during the summer and, then, in the winter, the supply is supplemented from the storage. Note that storage is also used as a reserve to reduce the impact of incidents. We model this difference using the following two scenarios:

Winter Reserves are depleted so any gas shortage must be immediately supplied from the network or from an alternative source.

Summer Gas shortages are compensated from the reserves, but these must be refilled, essentially converting a major supply shock into a smaller demand shock. For simplicity, we assume that refilling increases demand by $1 / 3$ of the shortage but with the fixed network. The alternative source is only 


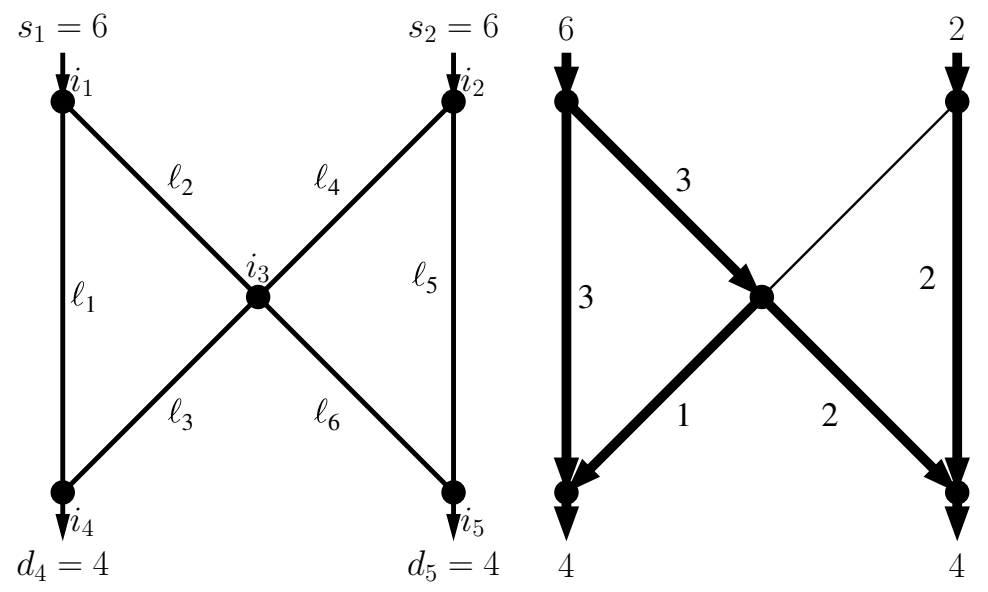

Figure 1: An example with 5 nodes and 6 pipelines and the optimal flow

used if this increased demand cannot be met.

\subsection{Example}

We consider the simple example presented in Figure 1 (left). This abstract example has 5 nodes and 6 pipelines connecting them. Two of these nodes are producers, supplying at most 6 units of gas, $p_{1}=300, p_{2}=400$, two are consumers, utilising 4 each. Node 3 has a balanced production/consumption pattern and each node has an alternative energy source at $\tilde{p}=600$.

The pipelines all have the same capacity $q=3$. We assume that transportation costs are negligible but positive, $C_{i j}=\varepsilon>0$ and, hence, will be ignored in the calculations but gas will always travel the shortest route in terms of pipeline segments.

For such a simple network, the optimal flows are easy to determine (Figure 1. right). Node $i_{4}$ obtains all the gas from the cheaper source $i_{1}$, so that the total cost is $c_{4}=4 \times 300=1200$. Node $i_{5}$ gets 2 from source $i_{1}$ and the rest from the more expensive $i_{2}$ at a total cost of $c_{5}=2 \times 300+2 \times 400=1400$.

Now, let us look at a possible disruption. If pipeline $\ell_{1}$ cannot be used, the flows change drastically. Now supplier $i_{1}$ is bound by the outgoing transmission capacity 3 via pipeline $\ell_{2}$; similarly, consumer $i_{4}$ can only obtain 3 from the network via $\ell_{3}$. 


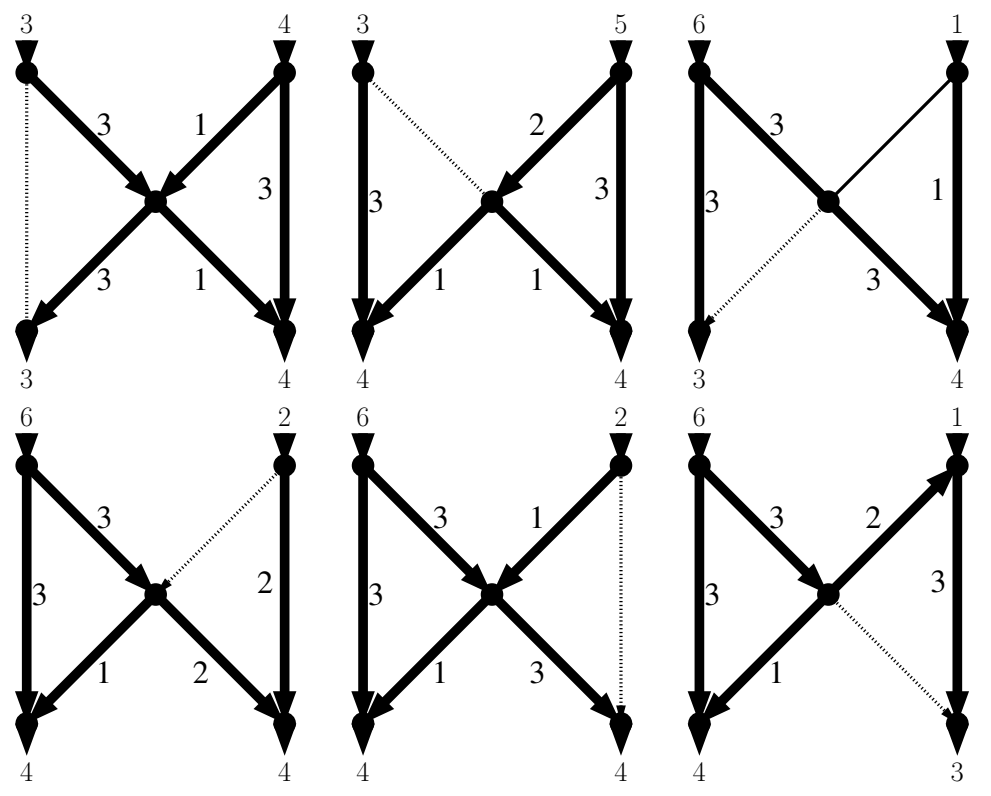

Figure 2: Optimal flows for disruptions in the winter (disrupted lines are dotted)

What are the costs? Consumer $i_{4}$ obtains all his gas via $i_{3}$. But is this gas from source $i_{1}$ or, at least in part, from $i_{2}$ ? By our assumption, the gas streams from the two sources are mixed, so from $i_{3}$, there are 4 units of gas available at a price calculated as the weighted average of the inflows: $p_{3}=\frac{3 \times 300+1 \times 400}{4}=325$.

In the winter scenario, consumer $i_{4}$ obtains 3 units of this gas as well as the remaining 1 supplied from its alternative source at a cost of 600: $c_{4}=$ $3 \times 325+1 \times 600=1575$, while $i_{5}$ has $c_{5}=1 \times 325+3 \times 400=1525$.

In the summer scenario, consumer $i_{4}$ can try to fill the storage in the coming 3 months. This results in a modified demand of $4 \frac{1}{3}$.

Focusing on the winter scenario, we can now calculate the costs of supply for all single-pipeline disruption scenarios (Table 1). Given the small number of states, calculating the expected shortfall (ES) with $\alpha=10 \%$ would be the same as looking at the worst case; instead, we consider $\alpha=\frac{1}{3}$, in other words, the average cost under the two worst scenarios (highlighted in Table 11). Therefore, the $\mathrm{ES}_{\frac{1}{3}}$ for the two nodes are 1537.5 and 1600 respectively, which constitute a $28 \%$ and $14 \%$ increase with respect to the status quo. Ostensibly, $i_{4}$ can be hurt more by losing its privileged connection to producer $i_{1}$ and a network disruption may affect $i_{4}$ more severely according to our model. 


\begin{tabular}{lcccc} 
& \multicolumn{2}{c}{ cost for node } & \multicolumn{2}{c}{ without $\ell_{4}$} \\
disrupted & $i_{4}$ & $i_{5}$ & $i_{4}$ & $i_{5}$ \\
\hline none & 1200 & 1400 & 1200 & 1400 \\
$\ell_{1}$ & $\mathbf{1 5 7 5}$ & 1525 & $\mathbf{1 7 2 5}$ & 1575 \\
$\ell_{2}$ & 1300 & $\mathbf{1 6 0 0}$ & $\mathbf{1 5 0 0}$ & $\mathbf{1 8 0 0}$ \\
$\ell_{3}$ & $\mathbf{1 5 0 0}$ & 1300 & 1500 & 1300 \\
$\ell_{4}$ & 1200 & 1400 & 1200 & 1400 \\
$\ell_{5}$ & 1225 & 1575 & 1275 & 1725 \\
$\ell_{6}$ & 1200 & $\mathbf{1 6 0 0}$ & 1200 & $\mathbf{1 8 0 0}$ \\
\hline ES $(\alpha=1 / 3)$ & 1537.5 & 1600 & 1612.5 & 1800
\end{tabular}

Table 1: Costs for $i_{4} \& i_{5}$ in the winter scenario. The worst $\frac{1}{3}$ are highlighted. The two right columns show the case when the network does not contain $\ell_{4}$. Note that the disruption of $\ell_{3}$ benefits $i_{5}$.

Such comparison is more useful when different networks are considered. In the initial network, pipeline $\ell_{4}$ carries no gas, deeming it unnecessary. The last two columns of Table 1 present the same analysis for a network with only 5 pipelines. It is clear that $\ell_{4}$ plays an important role in increasing supply security by mitigating the risks associated with disruptions. Without $\ell_{4}$, the expected shortfalls are 1612.5 and 1800 with a total increase of 275 . While one must compare this with the construction costs to see if $\ell_{4}$ is worth the money, it is clear that the pipeline is not useless even if it is not used - such as the Slovakia-Hungary interconnector at Beregdaróc (Badida, 2014).

\subsection{Scenarios}

Barring the baseline scenario, we consider certain possible developments for the Eastern corridor.

1. The "Ukraine" scenario assumes that the connection between Ukraine and Russia is closed, prompting the investigation into the effect of another closure.

2. In the second scenario, we calculate using a completed Nord Stream 2, 
3. in the third, the Ukraine-Russia pipeline is phased out once NS2 is operational.

For each of these scenarios, we have also looked at the effect of the opening of the Trans-Adriatic Pipeline connecting Turkey with Italy via Greece with a capacity of $20 \mathrm{bcm} / \mathrm{yr}$, carrying cheap gas from Central Asia to the South Balkans, Italy and beyond.

Generally, the construction of NS2 should alleviate the problem of congestion on the network, but Gazprom's communication about the Ukrainian pipelines reaching the end of their service life and the cost of maintaining excessive transmission capacities hints that the two main connections between Russia and Europe will not coexist for long. We, therefore, think that the impact of NS2's construction is best illustrated by comparing the first (Baseline) and last (Combined) scenarios. This will have a drastic effect on Eastern Europe and will incite interest in seeing whether TAP (and the already operational Trans-Anatolian Pipeline or TANAP) can compensate for the shift of the main East-West transmission channel to the North. The ongoing development of Balkan Stream will be an important contribution here.

In the following section, we present the data and results.

\section{Data and calculations}

The network of international pipelines form a connected network but, often, only via the national pipeline networks. We, therefore, use a number of simplifying assumptions (See also Csercsik, Hubert, Sziklai, and Kóczy, 2019)

- Players are reduced to a node; the connecting arcs, where necessary, include some segments of the national network.

- In the case of Russia and North Africa, distances are measured from the borders. This makes no difference for delivery prices.

- LNG is added as a player with a pipeline of the same length as the liquifying-regasification costs.

- Prices are estimates and the same for all consumers. 
- The cost of the alternative energy source is estimated at a flat $600 \mathrm{M} \$ / \mathrm{bcm}$.

- Differences in gas quality are ignored.

Data concerning national resources including natural gas are published regularly by a number of reliable sources. Transmission capacities were compiled from the data sheet provided by the International Energy Agency (2020). Consumption and production data was gathered from BP's Statistical Review of World Energy $(\overline{\mathrm{BP}}, 2020)$. Pipeline lengths were retrieved from the public database of the European Network of Transmission System Operators for Gas (ENTSOG, 2019) and other online sources.

\section{Results}

Our measure for supply security is based on the evaluation of the network with disrupted pipelines. We assume that all 150 pipelines face the same probability of incidents and for each country, we are concerned with the worst $10 \%$ of the cases, that is, the 15 (directed) pipelines whose removals give the highest cost increases for the country at hand. 
Table 2: Expected shortfall values for the different network scenarios in winter (remaining values are constant over all scenarios). TAP and NS2 stand for a scenario where the Trans-Adriatic Pipeline and Nord Stream 2 are, respectively, operational, UA for the one where Russia-Ukraine pipelines are closed.

\begin{tabular}{lrrrrrr} 
& base & TAP & NS2 & NS2+TAP & NS2+UA & \multicolumn{1}{l}{ All } \\
\hline Austria & 2110 & 2110 & 2027 & 2027 & 4740 & 4740 \\
Belgium & 10440 & 10302 & 5211 & 5215 & 5100 & 5100 \\
Bulgaria & 954 & 835 & 954 & 835 & 1940 & 1096 \\
Croatia & 232 & 232 & 232 & 232 & 532 & 532 \\
Czech Republic & 2098 & 2098 & 2095 & 2095 & 2318 & 2318 \\
France & 26023 & 26023 & 20107 & 19111 & 25836 & 25836 \\
Germany & 21527 & 21542 & 20721 & 20738 & 23904 & 23904 \\
Greece & 2629 & 1755 & 2629 & 1755 & 3058 & 1736 \\
Hungary & 2032 & 2033 & 2032 & 2033 & 4817 & 4618 \\
Italy & 31092 & 27151 & 30263 & 26898 & 38385 & 38385 \\
Netherlands & 3569 & 3569 & 2387 & 2389 & 2282 & 2282 \\
Poland & 4541 & 4505 & 4456 & 4456 & 5255 & 5255 \\
Romania & 303 & 304 & 303 & 304 & 720 & 473 \\
Serbia & 647 & 647 & 647 & 647 & 1276 & 1276 \\
Slovak Republic & 1169 & 1169 & 1169 & 1169 & 2747 & 2747 \\
Slovenia & 237 & 237 & 237 & 237 & 541 & 541 \\
Spain & 19030 & 19030 & 19030 & 19030 & 19030 & 19030 \\
Switzerland & 1724 & 1574 & 1083 & 1094 & 1149 & 1149 \\
Turkey & 11996 & 10843 & 11996 & 10843 & 13342 & 11643 \\
Ukraine & 2042 & 2042 & 2042 & 2042 & 4283 & 4251 \\
United Kingdom & 23520 & 23520 & 22099 & 22099 & 23520 & 23520
\end{tabular}


Table 3: Expected shortfall values for the different network scenarios in summer (remaining values are constant over all scenarios). As before, TAP and NS2 stand for a scenario where the Trans-Adriatic Pipeline and Nord Stream 2 are, respectively, operational, UA for the one where Russia-Ukraine pipelines are closed.

\begin{tabular}{lrrrrrr} 
& base & TAP & NS2 & NS2+TAP & NS2+UA & All \\
\hline Austria & 2235 & 2235 & 2112 & 2112 & 4940 & 4940 \\
Belgium & 10440 & 10440 & 5208 & 5210 & 5100 & 5100 \\
Bulgaria & 1050 & 840 & 1050 & 840 & 1956 & 1105 \\
Croatia & 379 & 379 & 379 & 379 & 870 & 870 \\
Czech Republic & 2140 & 2140 & 2116 & 2116 & 2342 & 2342 \\
France & 26029 & 26029 & 21769 & 21714 & 25287 & 25287 \\
Germany & 22150 & 22201 & 21209 & 21230 & 24377 & 24377 \\
Greece & 2601 & 1523 & 2430 & 1523 & 3058 & 1503 \\
Hungary & 2182 & 2183 & 2182 & 2184 & 5171 & 5171 \\
Italy & 28463 & 23879 & 25413 & 22488 & 34355 & 34239 \\
Netherlands & 4316 & 4293 & 4555 & 4559 & 4219 & 4218 \\
Poland & 4993 & 4993 & 4781 & 4869 & 5762 & 5762 \\
Romania & 1123 & 1123 & 1123 & 1123 & 2660 & 2127 \\
Serbia & 707 & 707 & 707 & 707 & 1393 & 1393 \\
Slovak Republic & 1177 & 1177 & 1177 & 1177 & 2766 & 2766 \\
Slovenia & 238 & 238 & 237 & 238 & 542 & 542 \\
Spain & 18902 & 18630 & 18775 & 18436 & 19030 & 19030 \\
Switzerland & 1776 & 1776 & 1132 & 1142 & 1151 & 1151 \\
Turkey & 12443 & 11137 & 12452 & 11137 & 13418 & 11685 \\
Ukraine & 3593 & 3593 & 3593 & 3593 & 8420 & 8420 \\
United Kingdom & 22195 & 22140 & 19273 & 17726 & 22148 & 22141
\end{tabular}



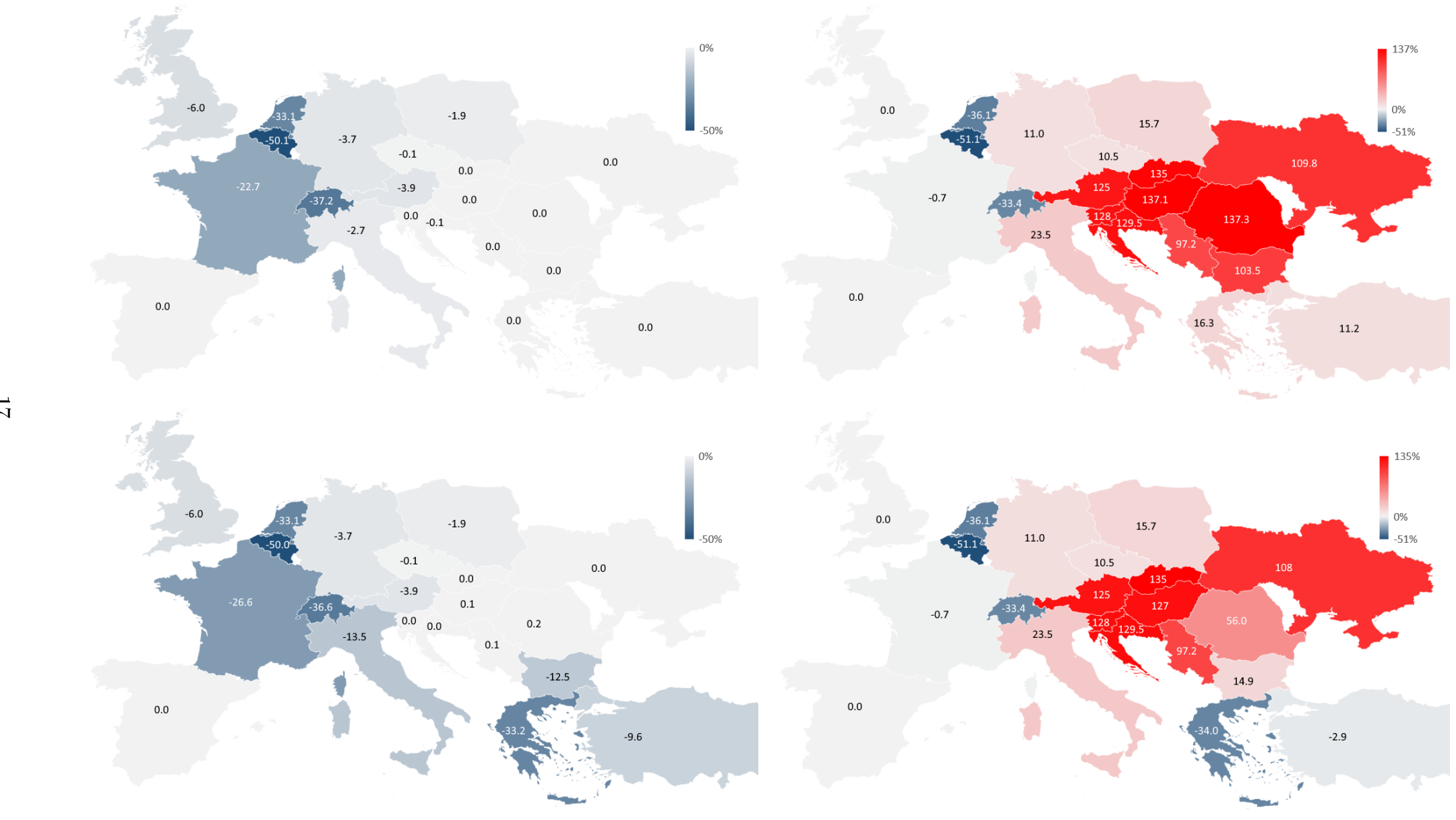

Figure 3: The winter scenarios: Nord Stream 2 (left), this without Ukraine (right), both with TAP (bottom row) as compared to the status quo 

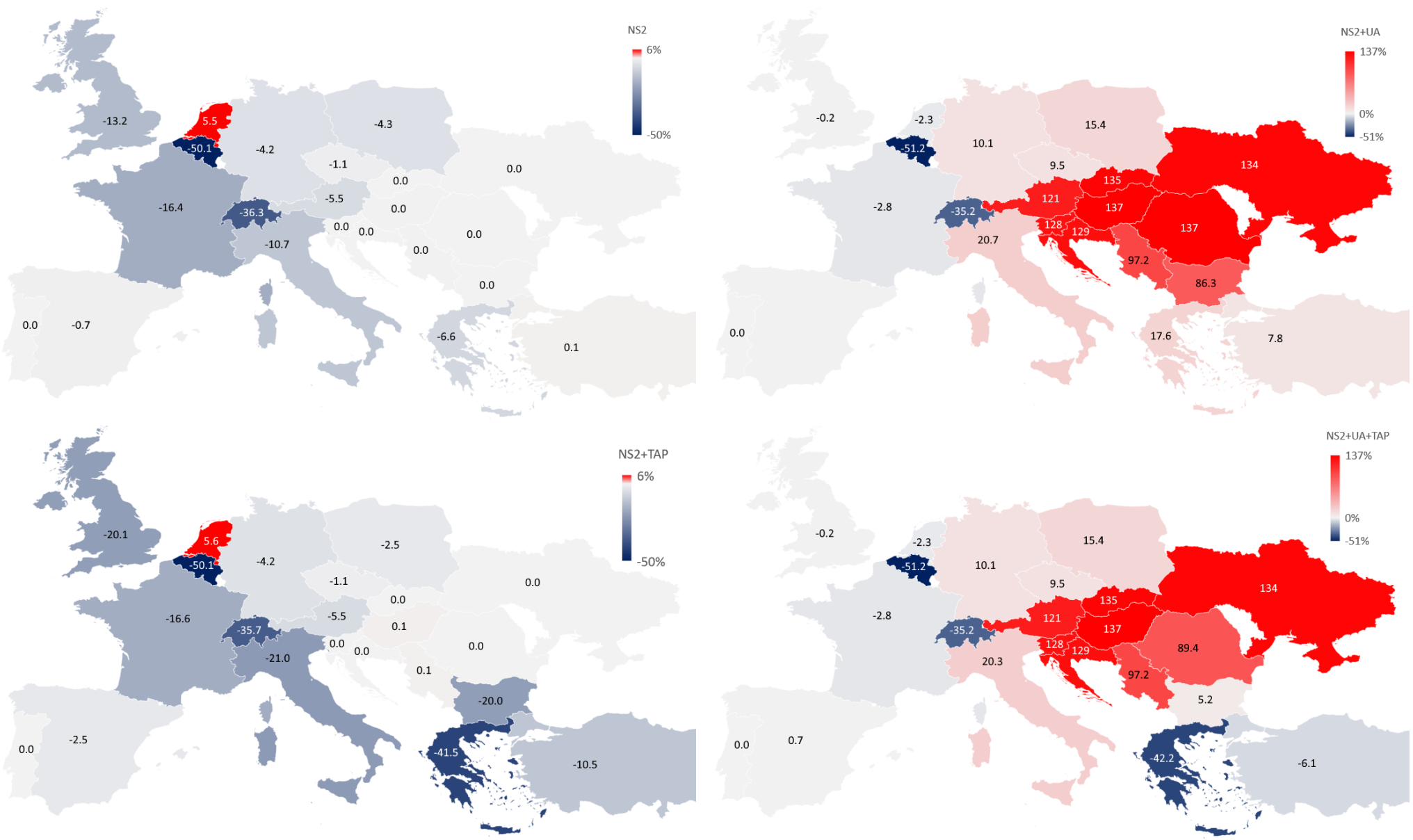

Figure 4: The summer scenarios: Nord Stream 2 (left), this without Ukraine (right), both with TAP (bottom row) as compared to the status quo 
We observe a drastic difference in the potential effects of NS2 in two parts of Europe: the East and West. While the construction of NS2 brings cheap gas and thereby substantial benefits to Western Europe, South-Eastern Europe enjoys no benefit at all. If NS2 is followed by phasing out the Ukrainian gas corridor, most of Central- and Eastern Europe is left with a highly concentrated supply path and, if any of the pipelines along that path are affected, the results will be catastrophic for the region, with substantial gas outages a surety. Interestingly, shortages are not restricted to the winter scenario. These risks, by far, outweigh the benefits of lower transportation costs for the West.

The Trans-Adriatic Pipeline system alleviates the problem in the Southern part of Europe but the current plans do not extend the network to the North benefiting only the few countries en route: Bulgaria, Greece and Italy. For some of these countries, the pipeline gives clear benefits vis-a-vis the status quo regarding supply security.

How far do the benefits of TAP reach? The average marginal contribution of TAP to risk is the highest for Greece and Bulgaria (-42 and $-39 \%)$, high for Romania, Turkey and Italy $(-21.4,-11.4$ and $-8.4 \%)$ and hits Poland most adversely at $0.16 \%$.

A similar analysis for NS2 depends heavily on whether it is an additional pipeline or there is a shift in existing connections to the North. If the former is true, it brings massive benefits to Switzerland, Belgium, France, the Netherlands and the UK as well as reduces risks in Italy, Austria, Germany and Poland. The highest losses will strike Hungary, Serbia, and Turkey at $0.02 \%$. On the other hand, if NS2 comes in a bundle, disruptions may create massive shortages in Slovakia, Hungary, Croatia, Slovenia, Austria, Ukraine, Romania and Serbia, additionally harming Bulgaria, Italy, Poland and, to a smaller extent, Germany, the Czech Republic and Turkey, creating savings in Belgium, the Netherlands, Switzerland and France only.

These results are generally in line with the division between supporters and opponents of the project (see, for instance, de Jong et al. 2020). Note that Germany and Austria face increased risks as well, their supportive stance can be explained by their special roles as distributing hubs for Eastern Europe under these scenarios. This is better modelled by a cooperative game theory approach 
(Sziklai, Kóczy, and Csercsik, 2020).

\section{Conclusion}

We have looked at the negative effect of the cost of disruptions in the system for each player. Our approach is very simple: by taking the same probability, we ignore differences in political, environmental and terrorist risks or even in the size or significance of a pipeline. Given the enormous observed differences in supply risk, we are confident in having found the weak links. Concerning the methodology: The Value at Risk (VaR) and Expected Shortfall methods are the two most commonly applied risk measures. We chose the latter since, in contrast to VaR, it is a coherent measure (Acerbi and Tasche, 2002); furthermore, VaR has been heavily criticised recently, see e.g. (Embrechts, 2000) or (Sarykalin et al. 2014). Heckmann et al. (2015) offer a comprehensive review on measuring risks related to supply chains.

At the time of writing, it is not entirely clear yet whether Nord Stream 2 can actually be completed and politics may affect its operation later, too. Most likely, however, the pipeline will get completed with the described effects. We must realise, however, that network disruptions also harm Russia: the shortages mean less gas sold for Russia. Russia has already made steps to contain the damages with the construction of TurkStream and Balkan Stream. Whether these steps are sufficient to compensate the region, we plan to investigate in another paper.

\section{Acknowledgement}

Our paper has benefited from extensive comments from Csaba Weiner and discussions with Bert Willems. This work has been supported by the grants $\mathrm{K}$ 131545 (Csercsik), K 128573 (Kóczy) and K 119930 (Sziklai) of the Hungarian National Research, Development and Innovation Office as well as the Higher Education Institutional Excellence Program 2020 of the Ministry of Innovation and Technology in the framework of the 'Financial and Public Services' research project (TKP2020-IKA-02) at Corvinus University of Budapest. Dávid Csercsik 
is a grantee of the János Bolyai Research Scholarship of the Hungarian Academy of Sciences.

\section{References}

Acerbi, C., 2002, Spectral measures of risk: A coherent representation of subjective risk aversion, Journal of Banking \& Finance 26, 1505-1518.

Acerbi, C. and D. Tasche, 2002, On the coherence of expected shortfall, Journal of Banking \& Finance 26, 1487-1503.

Adam, A., M. Houkari, and J.-P. Laurent, 2008, Spectral risk measures and portfolio selection, Journal of Banking \& Finance 32, 1870 - 1882.

Adomaitis, N. and T. Solsvik, 2020, Six Norway oil and gas fields shut as strike escalates.

Badida, J., 2014, Changing European gas map - an opportunity or threat for Slovakia?, International Issues \& Slovak Foreign Policy Affairs 23, 3-11.

Bartelet, H. and M. Mulder, 2020, Natural gas markets in the European Union: Testing resilience, Economics of Energy \& Environmental Policy 9.

Basel Committee on Banking Supervision, 2013, Fundamental review of the trading book: A revised market risk framework, Consultative document.

Biresselioglu, M. E., T. Yelkenci, and I. O. Oz, 2015, Investigating the natural gas supply security: A new perspective, Energy 80, 168-176.

BP, 2020, Statistical review of world energy 2020 (London, UK).

Cabalu, H., 2010, Indicators of security of natural gas supply in Asia, Energy Policy 38, 218-225.

Csercsik, D., F. Hubert, B. R. Sziklai, and L. A. Kóczy, 2019, Modeling transfer profits as externalities in a cooperative game-theoretic model of natural gas networks, Energy Economics 80, 355-365.

Denault, M., 2001, Coherent allocation of risk capital, Journal of Risk 4, 1-39. 
Doukas, H., A. Flamos, and J. Psarras, 2011, Risks on the Security of Oil and Gas Supply, Energy Sources, Part B: Economics, Planning, and Policy 6, $417-425$.

Embrechts, P., 2000, Extreme value theory: Potential and limitations as an integrated risk management tool, Derivatives Use, Trading \& Regulation 6 , 449-456.

Energy Compass, 1997, Bomb disrupts algerian gas supplies , 21-23.

ENTSOG, 2019, The European Natural Gas Network, Retrieved from https://www.entsog.eu/sites/default/files/2020-01/ENTSOG_CAP_ 2019_A0_1189x841_FULL_401.pdf, Accessed: 2020-04-08.

Gas Matters, 2001, Arun challenges Asian LNG reliability rules, Gas Matters, $1-6$.

Hassel, A. and C. Egenhofer, 2017, Lessons from the Baumgarten incident, CEPS Commentary , 11-12.

Heckmann, I., T. Comes, and S. Nickel, 2015, A critical review on supply chain risk - Definition, measure and modeling, Omega 52, 119 - 132.

International Energy Agency, 2020, Gas Trade Flows, Retrieved from https: //www.iea.org/reports/gas-trade-flows, Accessed: 2020-04-08.

Jirušek, M., 2020, The attitude of the visegrad group countries towards russian infrastructural projects in the gas sector, Energy Policy 139, 111340.

de Jong, M., T. Van de Graaf, and T. Haesebrouck, 2020, A matter of preference: Taking sides on the Nord Stream 2 gas pipeline project, Journal of Contemporary European Studies, 1-14.

Kruyt, B., D. P. van Vuuren, H. J. de Vries, and H. Groenenberg, 2009, Indicators for energy security, Energy Policy 37, 2166-2181.

Lu, W., M. Su, B. D. Fath, M. Zhang, and Y. Hao, 2016, A systematic method of evaluation of the Chinese natural gas supply security, Applied Energy 165, 858-867. 
Majidi-Qadikolai, M. and R. Baldick, 2016, Integration of $N-1$ contingency analysis with systematic transmission capacity expansion planning: ERCOT case study, IEEE Transactions on Power Systems 31, 2234-2245.

Pavlović, D., E. Banovac, and N. Vištica, 2018, Defining a composite index for measuring natural gas supply security - the croatian gas market case, Energy Policy $114,30-38$.

Pirani, S., J. Stern, and K. Yafimava, 2009, The Russo-Ukrainian gas dispute of January 2009: a comprehensive assessment, vol. NG 27.

Praks, P., V. Kopustinskas, and M. Masera, 2015, Probabilistic modelling of security of supply in gas networks and evaluation of new infrastructure, Reliability Engineering \& System Safety 144, $254-264$.

Sarykalin, S., G. Serraino, and S. Uryasev, 2014, Value-at-Risk vs. Conditional Value-at-Risk in risk management and optimization, chap. 13, 270-294.

Scotti, M. and B. Vedres, 2012, Supply security in the European natural gas pipeline network, in: M. Scotti and B. Vedres, eds., Networks in Social Policy Problems (Cambridge University Press), 237-264.

Stern, J., 2002, Security of European natural gas supplies: the Impact of Import Dependence and Liberalization, The Royal Institute of International Affairs , 36 .

Szegö, G., 2002, Measures of risk, Journal of Banking \& Finance 26, 1253-1272.

Sziklai, B. R., L. Kóczy, and D. Csercsik, 2020, The impact of Nord Stream 2 on the European gas market bargaining positions, Energy Policy 144, 111692.

Tarnawski, M., 2015, Security of Gas Supply in the Countries of the Visegrád Group, Securitologia 21, 127-142.

Tóth, B. T., P. Kotek, and A. Selei, 2020, Rerouting Europe's gas transit landscape - Effects of Russian natural gas infrastructure strategy on the V4, Energy Policy 146, 111748.

Vainio, J., 2017, An explosion in the heart of the European gas system: What does the Baumgarten case tell about the resiliency of the system?, SARDINES 5,7 . 
Vianello, C. and G. Maschio, 2014, Quantitative risk assessment of the italian gas distribution network, Journal of Loss Prevention in the Process Industries $32,5-17$.

Weisser, H., 2007, The security of gas supply-a critical issue for Europe?, Energy Policy $35,1-5$.

Wood, S. and O. Henke, 2021, Denmark and Nord Stream 2: A small state's role in global energy politics, Energy Policy 148, 111991.

World Gas Report, 1986, Norwegian gas customers unruffled by production shutdown, 3 . 